\begin{tabular}{|c|c|c|c|c|c|}
\hline MUNIBE Antropologia-Arkeologia & $n^{\circ} 72$ & $19-23$ & DONOSTIA & 2021 & ISSN 1132-2217 • eISSN 2172-4555 \\
\hline
\end{tabular}

\title{
Un bifaz del Paleolitico Medio localizado en el entorno del yacimiento de Aranbaltza (Barrika, Bizkaia)
}

\author{
A Middle Paleolithic handaxe found in the surroundings \\ of Aranbaltza site (Barrika, Bizkaia)
}

PALABRAS CLAVES: Tecnología Lítica, basalto, hallazgo, bifaz.

GAKO-HITZAK: Teknologia litikoa, basaltoa, aurkikuntza, aurpegibikoa.

KEY WORDS: Lithic tecnology, basalt, discovery, handaxe.

\section{Joseba RIOS-GARAIZAR ${ }^{(1)}$, Aixa SAN EMETERIO(2), Alesander TREBOLAZABALA(3)}

\section{RESUMEN}

Se presenta el hallazgo de un bifaz de basalto en Lastarri, cerca del yacimiento de Aranbaltza. Se analizan sus características morfo-tecnológicas en relación con los bifaces atribidos al Paleolítico Inferior y Medio en el área cantábrica del País Vasco y se concluye que probablemente este bifaz está relacionado con las ocupaciones del Paleolítico Medio de Aranbaltza (120-55.000 años).

\section{LABURPENA}

Lastarrin, Aranbaltza aztarnategitik hurbil, aurkitu dugun basaltozko aurpegibikoa aurkeztu dugu. Ezaugarri morfo-teknologikoak aztertu ditugu, Euskal Herriko kantauriar isurialdeko eremuetako Behe- eta Erdi-Paleolitoko aurpegibikoen arabera, eta ondorioztatu dugu aurpegibiko hau ziurrenik Aranbaltzako Erdi-Paleolitoko egonlekuekin lotuta dagoela (120-55.000 urte).

\section{ABSTRACT}

We present the discovery of a basalt handaxe made in Lastarri, close to the site of Aranbaltza. Also, we analyze its morpho-technological features in comparison with other bifaces attributed to the Lower and Middle Paleolithic in the cantabrian area of the Basque Country. We conclude that, probably, this handaxe is related to the Middle Paleolithic occupations of Aranbaltza (120-55.000 years).

\section{INTRODUCCIÓN}

En el marco de las investigaciones arqueológicas del yacimiento de Aranbaltza, iniciadas en el año 2013 se han realizado diversas prospecciones superficiales en su entorno. En 2018 el miembro del equipo de excavación, Alesander Trebolazabala, localizó un bifaz de basalto en el lecho del arroyo Urgozo, junto a las ruinas del molino de Lastarri.

Los hallazgos de esta naturaleza, sin contexto arqueológico preciso, son siempre difíciles de evaluar, más aún teniendo en cuenta que los bifaces son herramientas comunes en contextos del Pleistoceno Medio (Paleolítico Inferior o Paleolítico Medio Antiguo), pero también al final del Paleolítico Medio e incluso, aunque de manera mucho más esporádica, en el Paleolítico Superior. La cercanía del lugar del hallazgo al complejo arqueológico de Aranbaltza sugiere que esta pieza pudo formar parte de este registro arqueológico. En esta nota presentamos una descripción pormenorizada del resto y lo ponemos en relación con las ocupacio- nes del Paleolítico Medio de Aranbaltza (Rios-Garaizar et al., 2018) y con otras evidencias semejantes del entorno inmediato del yacimiento (Rios-Garaizar et al., 2012a; 2013).

\section{CONTEXTO}

El bifaz fue localizado en el cauce del arroyo Urgozo, junto a las ruinas del molino de Lastarri, justo en el punto en el que se unen los dos cauces que nacen a ambos lados del collado de Ametzaga y que conforman el arroyo, que en su ramal occidental drena el yacimiento de Ollagorta-Aranbaltza. La zona de Ollagorta y Aranbaltza fue explotada como cantera de áridos en las décadas de 1950 y 1960, y el entorno conserva las severas huellas de esta actividad en forma de taludes, escombreras, circos de cantera, canalizaciones, presillas, etc. Afortunadamente la afección provocada por esta actividad extractiva en el yacimiento de Aranbaltza no fue total permitiendo la conservación de parte del yacimiento. En la década de los 50 se recuperaron

\footnotetext{
(1) Bizkaiko Arkeologi Museoa, Mallona Galtzada, 2, 48006 Bilbo, Bizkaia jorios76@gmail.com

(2) aixitaseg@gmail.com

(3) alestrebo@hotmail.com
} 


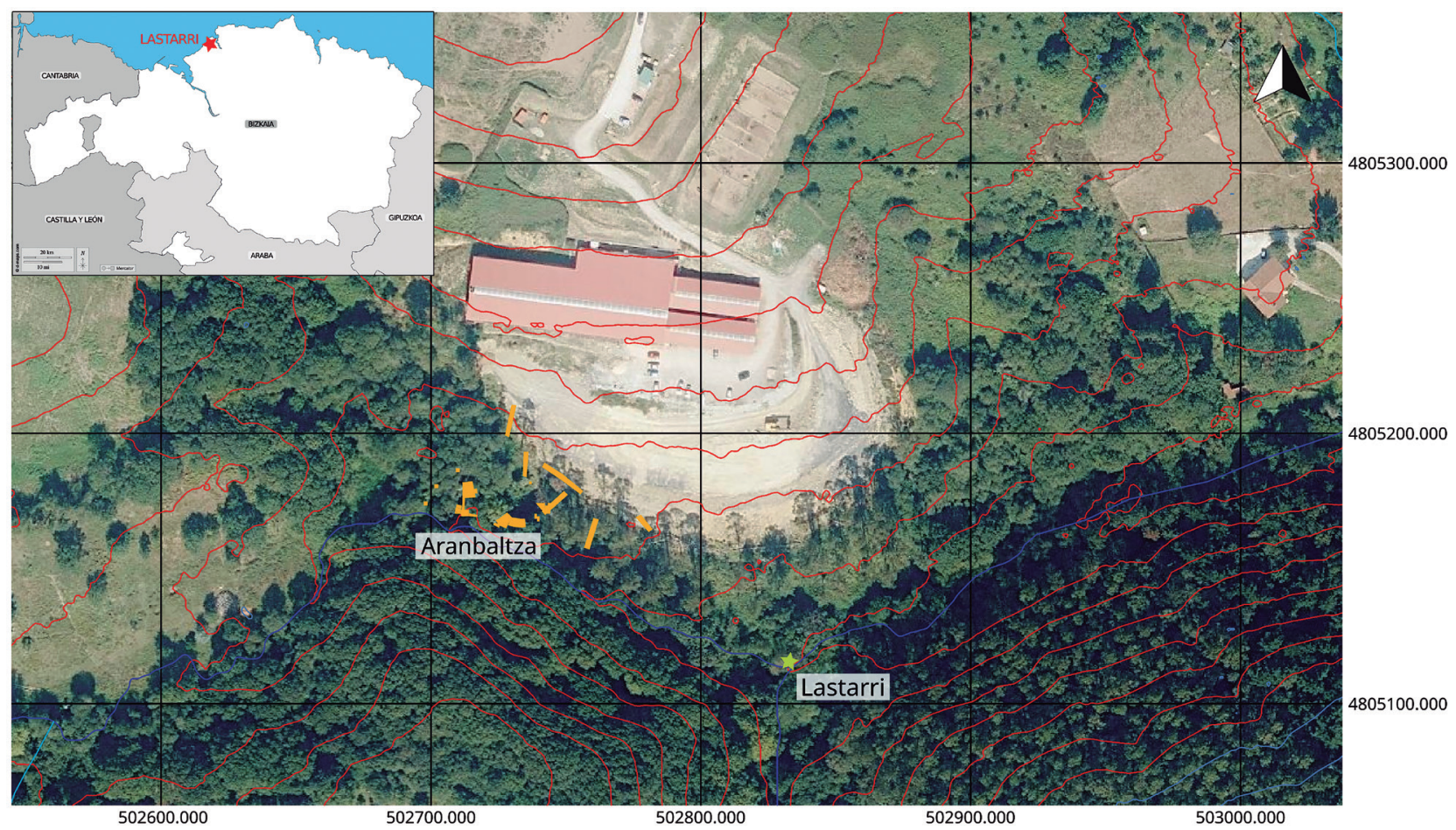

Fig.1. Mapa con la localización del punto donde se encontró el bifaz de Lastarri con respecto al yacimientno de Aranbaltza (cuadros de 100 m de lado) / Map with the position of the finding place of Lastarri handaxe in relation with the Aranbaltza site (squares have 100m sides)

numerosos materiales en la cantera de Ollagorta-Aranbaltza y en 1959 se realizaron dos sondeos que revelaron la existencia de un gran yacimiento arqueológico al aire libre (Barandiarán Ayerbe et al., 1960). Posteriormente, en la década de los 2000, se realizaron trabajos de canalización de agua que afectaron al yacimiento provocando la destrucción de parte de sus depósitos (Rios-Garaizar et al., 2012b; 2020).

En su paso junto a Aranbaltza el arroyo Urgozo corta y erosiona los depósitos de arena y arcilla del yacimiento, arrastrando materiales arqueológicos que ocasionalmente se pueden localizar en el lecho del arroyo, rio abajo. Además, en el entorno del molino en ruinas se ha detectado los restos de una pequeña presa de cemento que debió estar en funcionamiento hasta épocas recientes y que, en su parte posterior, acumuló sedimentos arenosos y gravas, probablemente producto de la erosión asociada a la explotación de la cantera. Parece razonable, por tanto, pensar que el bifaz de Lastarri pudo ser arrastrado desde el yacimiento de Aranbaltza hasta el punto donde se produjo el hallazgo. En otros puntos del cauce del Urgozo, rio abajo de Aranbaltza, es frecuente encontrar restos líticos, incluyendo algunos núcleos Levallois muy característicos.

\section{DESCRIPCIÓN DEL BIFAZ}

Se trata de un bifaz ovalado de pequeñas dimensiones (Tabla I) fabricado a partir de un canto rodado aplanado con una faceta plana natural en un lateral.
Hemos podido identificar la materia prima como un basalto de grano fino con un alto contenido en hierro y numerosas vacuolas. Actualmente la pieza tiene una coloración gris verdosa, con zonas rojizas, pero en los desconchados recientes de la parte distal se puede observar el color negro original de la roca. Aunque el origen del basalto no ha podido ser establecido con precisión, la faceta plana natural puede interpretarse como un plano de disyunción columnar, lo que nos situaría un origen probable en la formación de basaltos columnares de Fruiz. El hecho de que la pieza tenga gran parte de su superficie exterior rodada indica que el material fue extraído de un contexto aluvial secundario. El estado de conservación de la pieza es muy bueno, con la excepción de algunos desconchados en el filo distal y en el filo izquierdo, la pieza no muestra alteraciónes mecánicas reseñables, estando las aristas muy ligeramente erosionadas.

\begin{tabular}{|l|c|}
\hline Peso & $324 \mathrm{~g}$ \\
\hline $\mathbf{L}$ & $101,43 \mathrm{~mm}$ \\
\hline $\mathbf{M}$ & $74,66 \mathrm{~mm}$ \\
\hline $\mathbf{E}$ & $37,22 \mathrm{~mm}$ \\
\hline $\mathbf{a}$ & $55,88 \mathrm{~mm}$ \\
\hline $\mathbf{n}$ & $70,68 \mathrm{~mm}$ \\
\hline $\mathbf{o}$ & $56,25 \mathrm{~mm}$ \\
\hline $\mathbf{L} \mathbf{a}$ & 4,33 \\
\hline $\mathbf{n} / \mathbf{m}^{*} \mathbf{1 0 0}$ & 91,8 \\
\hline
\end{tabular}

Tabla 1: Carácterísticas métricas de bifaz de Lastarri. / Metric features of Lastarri handaxe. 

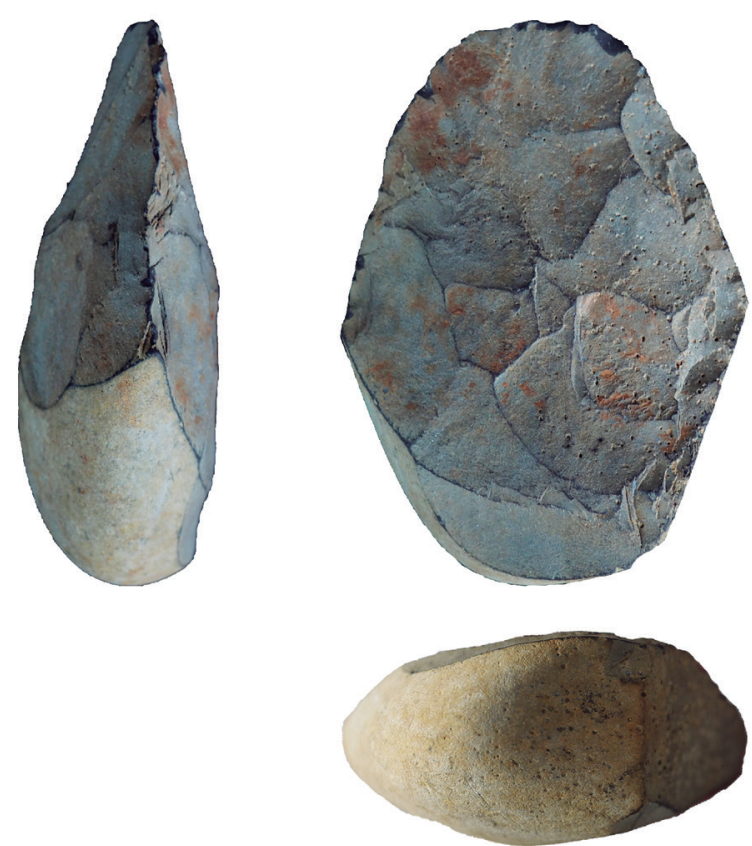

Fig.2. Bifaz de Lastarri / Lastarri handaxe.

El bifaz se configura en los dos tercios distales, mediante grandes lascados invasores, quedando reservada la zona proximal con neo-córtex. La cara superior está rebajada mediante series de lascados invasores desde los laterales, mientras que en la cara inferior el desarrollo de los lascados es convergente, quedando más superficie neocortical reservada. La morfología resultante es ovalar, con un perfil longitudinal triangular, un perfil transversal ovalado y un filo distal convexo. El borde derecho está ocupado en una mitad por el plano de fractura natural, mientras que el filo, recto, presenta desconchados bifaciales que pueden ser producto de un uso en percusión cortante, o bien un intento de "matar" el filo para crear una superficie de prensión. El filo izquierdo, algo más sinuoso, presenta pequeños lascados alternantes que pueden estar producidos también por el uso en en percusión cortante. El extremo distal y el filo izquierdo presentan desconchados recientes que han afectado parcialmente al filo.

\section{TECNOLOGÍA BIFACIAL EN EL PALEOLÍTICCO MEDIO ANTIGUO DEL ORIENTE DE LA REGIÓN CANTÁBRICA}

Los yacimientos del Oriente de la Región Cantábrica que han proporcionado bifaces son muy escasos y de naturaleza dispar. Los únicos recuperados en contexto arqueológico preciso son los del yacimiento de Lezetxiki (Baldeón, 1993), Amalda I (Rios-Garaizar, 2012), Irikaitz (Arrizabalaga e Iriarte, 2004) y Arlanpe (Rios-Garaizar, 2013). Además hay bifaces recuperados fuera de contexto como Jaizkibel (Arrizabalaga, 1994), Arantzasagasti (inédito), Kurtzia (Barandiarán et al., 1960), Aretxabaleta, Mendibarrena, Ondiz, Diliz, Zientoetxe o
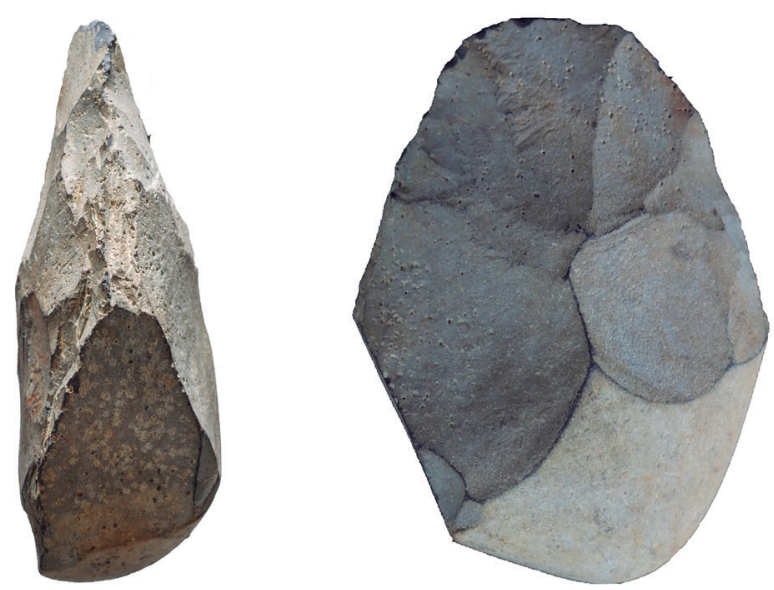

Errementariena (Rios Garaizar et al., 2012b, 2013). En contextos cercanos hay bifaces provenientes de yacimientos como Koskobilo (Vallespí y Ruiz de Gaona, 1971), Urrunaga (Sáenz de Buruaga y Urigoitia, 1986, Fernández Eraso et al., 2017), Manzanos (Baldeón, 1990a) o Lumbier (Mensua y Marcos, 1958). Tradicionalmente, se ha considerado estos bifaces como ejemplos de tecnología achelense, y por tanto evidencias de un poblamiento durante el Paleolítico Inferior. Sin embargo, recientemente hemos podido certificar el uso de tecnología bifacial en momentos antiguos del Paleolítico Medio en combinación con industrias de lascas con tecnología Levallois, Discoide y SSDA en el yacimiento de Arlanpe (Rios-Garaizar, 2013; Rios-Garaizar et al. 2015) y probablemente en el nivel $V$ de Lezetxiki (Baldeón, 1993), así como la presencia de bifaces en el Musteriense final (MTA) de Amalda (Baldeón 1990b, Rios-Garaizar, 2010; 2012). Parece por tanto que el uso de bifaces no es exclusivo del Achelense sino que es también una manifestación típica de los repertorios industriales del Paleolítico Medio regional especialmente del Paleolítico Medio antiguo (Turq et al., 2010) y de tecnocomplejos como el Vasconiense o el MTA (Deschamps, 2017).

En el propio yacimiento de Aranbaltza se han recuperado piezas bifaciales en distintos contextos de Aranbaltza I y III. En Aranbaltza I se ha documentado un pequeño bifaz (63x 45x 16 mm) fabricado en una plaqueta de sílex, muy parecido a otro fabricado en sílex que fue recuperado en Kurtzia. En Aranbaltza III, por otro lado, se ha recuperado en un contexto alterado, pero muy probablemente proveniente de la US5-6, un canto rodado de basalto con cofiguración bifacial. También en Aranbaltza III, en 2019, se recuperó una gran pieza bifacial fabricada en traquita en la US6. 
Además, si comparamos los bifaces de Arlanpe, Lezetxiki y Amalda podemos observar ciertas caracterśiticas comunes, como el uso de rocas de grano fino (lutita, basalto y vulcanita) como material prima; la presencia de amplias superficies corticales, generalmente en la base; y la tendencia hacia una morfología ovalada. Muchos de los bifaces recuperados en superficie en Bizkaia, como por ejemplo el de Kurtzia (Barrika), los dos de Zientoetxe (Getxo), el de Aretxabaleta (Leioa) o el de Arantzasagasti (Dima) comparten estas características (Rios-Garaizar et al., 2012; 2013). El bifaz que presentamos en este trabajo tiene unas características análogas a los anteriormente mencionados, aunque es uno de los ejemplares más pequeños (unos 100 mm de longitud). Si tenemos en cuenta todo esto, y además constatamos que en Aranbaltza no se han documentado registros arqueológicos anteriores a 150.000 años, podemos proponer con cierta seguridad una atribución al Paleolítico Medio de este resto. Si corresponde al Paleolítico Medio Antiguo, documentado en las US5 y US6 de Aranbaltza III, o al Paleolítico Medio final de la US1 de Aranbaltza III o el nivel D de Aranbaltza I es, sin embargo, más difícil de precisar.

\section{AGRADECIMIENTOS}

Las investigaciones en el yacimiento de Aranbaltza se han realizado gracias a la colaboración desinteresada de Damián Líbano, propietario de los terrenos donde se encuentra el yacimiento; a la financiación de la Diputación Foral de Bizkaia (2013-2021), del Gobierno Vasco (2014-2015), de Harpea Kultur Elkartea (20132017) y de Edestiaurre Arkeologi Elkartea (2018), y a la colaboración del Ayuntamiento de Barrika (2014-2018). Todo este trabajo no habría podido ser realizado sin la colaboración de un nutrido grupo de voluntarios que desde 2013 han colaborado de manera desinteresada en las excavaciones. Finalmente, queremos tener un recuerdo especial para Iluminada Ortega que entre 2014 y 2018 ejerció como co-directora del proyecto y que desgraciadamente nos dejó en 2019.

\section{INFORMACIÓN SUPLEMENTARIA}

Modelo 3D de superficie del bifaz: (https://doi.org/10.6084/m9.figshare.17026085.v1).

\section{BIBLIOGRAFÍA}

Arrizabalaga, A., 1994. Hallazgo de un bifaz y otros restos liticos en el monte Jaizkibel (Hondarribia, Gipuzkoa). Munibe Antropologia-Arkeologia 46, 23-31.

Arrizabalaga, A., Iriarte Chiapusso, M.J., 2004. El yacimiento arqueológico de Irikaitz (Zestoa, País Vasco). Descripción del depósito y caracterización industrial de su nivel IV. En: Actas de la XI reunión Nacional de Cuaternario 2003. 205-210.

Baldeón, A., 1990a. El Paleolítico Inferior y Medio en el País Vasco. Una aproximación en 1990. Munibe Antropologia-Arkeologia 42, 11-22.
Baldeón, A., 1990b. La industria lítica de los niveles paleolíticos: En: Altuna, J., Baldeón, A., Mariezkurrena, K. (Eds.), La Cueva de Amalda (Zestoa, País Vasco). Ocupaciones Paleolíticas y Postpaleolíticas, 63-115. Eusko Ikaskuntza, San Sebastián.

Baldeón, A., 1993. El yacimiento de Lezetxiki (Gipuzkoa, Pais Vasco). Los niveles musterienses. Munibe Antropologia-Arkeologia 45, 3-97.

Barandiarán Ayerbe, J.M., Aguirre, A., Grande, M., 1960. Estación de Kurtzia (Barrica-Sopelana), Servicio de Investigaciones Arqueológicas de la Excma. Diputación Provincial de Vizcaya, Bilbao.

Deschamps, M., 2017. Late Middle Palaeolithic assemblages with flake cleavers in the western Pyrenees: The Vasconian reconsidered. Quaternary International 433 B, 33-49. https://doi. org/10.1016/j.quaint.2016.01.043

Fernández Eraso, J., García-Rojas, M., Sánchez, A., Prieto, A., Calvo, A., Domínguez-Ballesteros, E., Tarriño, A., López-deOcáriz, J., Bradtmöller, M., Urigoitia, T., 2017. El tecno-complejo del Embalse de Urrúnaga (Álava). Nuevas aportaciones al conocimiento de las sociedades del Paleolítico inferior en el norte de la Península Ibérica. Munibe Antropologia-Arkeologia 68, 5-31.

Marcos, A., Mensua, S., 1959. Un hallazgo lítico del Paleolítico inferior, del término de Lumbier (Navarra). Príncipe de Viana 76-77, 217-225.

Ríos Garaizar, J., 2010. Organización económica de las sociedades neanderales: el caso del nivel VII de Amalda (Zestoa, Gipuzkoa). Zephyrus LXV, 15-37.

Rios-Garaizar, J., 2012. Industria lítica y sociedad en la Transición del Paleolítico Medio al Superior en torno al Golfo de Bizkaia. PUbliCan - Ediciones de la Universidad de Cantabria, Santander.

Rios-Garaizar, J., 2013. Industria lítica de los niveles del Paleolítico Medio Antiguo y Paleolítico Superior de Arlanpe (Lemoa, Bizkaia). En: Rios-Garaizar, J., Garate Maidagan, D., Gómez-Olivencia, A. (Eds.), La Cueva de Arlanpe (Lemoa). Ocupaciones Humanas Desde El Paleolítico Medio Antiguo Hasta La Prehistoria Reciente, 177-253. Kobie Serie BAI 3. Diputación Foral de Bizkaia, Bilbao.

Rios-Garaizar, J., Libano Silvente, I., Garate Maidagan, D., 2012a. Nuevas localizaciones del Paleolítico Inferior en Uribe Kosta (Bizkaia): Los yacimientos de Moreaga (Sopelana) y Errementariena (Barrika). Kobie Paleoantropología 31, 45-56.

Ríos-Garaizar, J., Libano Silvente, I., Garate Maidagan, D., 2012b. El yacimiento chatelperroniense al aire libre de Aranbaltza (Barrika, Euskadi). Munibe Antropologia-Arkeologia 63, 81-92.

Rios-Garaizar, J., Libano Silvente, I., Garate Maidagan, D., Gómez-Olivencia, A., Regalado Bueno, E., 2013. Nuevas Iocalizaciones arqueológicas al aire libre del Paleolítico Inferior y Medio en las cuencas del Gobela, del Udondo (Bizkaia). Kobie Paleoantropología 32, 39-60.

Rios-Garaizar, J., Maidagan, D.G., Gómez-Olivencia, A., Iriarte, E., Arceredillo-Alonso, D., Iriarte-Chiapusso, M.J., Garcia-Ibaibarriaga, N., García-Moreno, A., Gutierrez-Zugasti, I., Torres, T., Aranburu, A., Arriolabengoa, M., Bailón, S., Murelaga, X., Ordiales, A., Ortiz, J.E., Rofes, J., Pedro, Z.S., 2015. Short-term Neandertal occupations in the late Middle Pleistocene of Arlanpe (Lemoa, northern Iberian Peninsula). Comptes Rendus - Palevol 14, 233-244. https://doi.org/10.1016/j.crpv.2014.11.006 
Rios-Garaizar, J., López-Bultó, O., Iriarte, E., Pérez-Garrido, C., Piqué, R., Aranburu, A., Iriarte-Chiapusso, M.J., Ortega-Cordellat, I., Bourguignon, L., Garate, D., Libano, I., 2018. A Middle Palaeolithic wooden digging stick from Aranbaltza III, Spain. PLOS ONE 13, e0195044. https://doi.org/10.1371/ journal.pone.0195044

Rios-Garaizar, J., San Emeterio, A., Iriarte, E., López-Bultó, O., Arnold, L.J., Bourguignon, L., Iriarte-Chiapusso, M.-J., Pérez-Garrido, C., Piqué, R., Campaña Lozano, I., Bermejo Albarrán, L., Sánchez-Romero, L., Demuro, M., Marín-Arroyo, A.B., Lahaye, C., Aranburu, A., Arranz-Otaegui, A., Cubas, M., Benito-Calvo, A., Garate, D., Gómez-Olivencia, A., Ortega, I., 2020. El complejo arqueológico de Aranbaltza (Barrika, Bizkaia). Una ventana a las formas de vida de los Neandertales fuera de las cuevas en la Región Cantábrica. En: Actualidad de la investigación arqueológica en España II, 29-44. Museo Arqueológico Nacional, Madrid.

Sáenz de Buruaga, J.A., Urigoitia, T., 1986. Evidencias aisladas de cantos tallados en las márgenes del embalse de Urrunaga (Alava). Cuadernos de Arqueología Alavesa 13, 29-46.

Turq, A., Brenet, M., Colonge, D., Jarry, M., Lelouvier, L.-A., O'Farrell, M., Jaubert, J., 2010. The first human occupations in southwestern France: A revised summary twenty years after the Abbeville/Saint Riquier colloquium. Quaternary International 223-224, 383-398.

Vallespí, E., Ruiz de Gaona, M., 1971. Piezas inéditas de tradición achelense en las series líticas de Coscobilo de Olazagutía (Navarra). Munibe 23, 375-384. 
